Introduction The development and use of nanoparticles for medical applications is rapidly increasing. One of the most commonly used nanoparticles is silver, due to its antimicrobial properties. At present the use of silver nanoparticles is unregulated and there is increasing concern regarding their potential adverse health effects, particularly when inhaled. The alveolar region is the primary site of nanoparticle deposition following inhalation, thus it is important to consider the effects they will exert on the alveolar epithelium, including potential toxicity. The alveolar epithelium is covered with a surfactant layer, acting as a barrier to inhaled particulate matter and pathogens, with which nanoparticles will interact before reaching the epithelium. Thus, we hypothesise that the surfactant layer will influence the toxicity and inflammatory response of alveolar epithelial cells to inhaled AgNPs.

Methods Immortalised human alveolar type 1 epithelial (TT1) cells were exposed to increasing concentrations of $20 \mathrm{~nm}$ or $110 \mathrm{~nm}$ silver nanoparticles (AgNP) in the presence and absence of porcine surfactant (Curosurf) for 24 hours. Cell viability was measured using the MTS assay and IL-6 and IL-8 release was measured by ELISA. In addition, the effect of the antixodiant glutathione on cytokine release was assessed.

Results Exposure to $20 \mathrm{~nm}$ and $110 \mathrm{~nm}$ AgNP did not significantly affect cell viability in the presence or absence of Curosurf $(0-50 \mu \mathrm{g} / \mathrm{ml})$. However, IL-6 and IL-8 release was significantly increased $(\mathrm{P}<0.0005)$ for all AgNP at concentrations above $10 \mu \mathrm{g} / \mathrm{ml}$. Furthermore, preincubation of AgNP with Curosurf significantly inhibited this response $(\mathrm{P}<0.001)$. Pre-treatment of cells with glutathione also significantly inhibited IL-6 and IL-8 release following AgNP exposure. When glutathione and Curosurf were combined there was a further inhibition of cytokine release.

Conclusion This study demonstrates that AgNP, whilst not overtly toxic, induce an inflammatory response in human alveolar epithelial cells that is driven by oxidative stress. Furthermore, we have shown that the presence of surfactant significantly attenuates the inflammatory response suggesting a protective effect against inhaled nanoparticles. Thus, this study demonstrates the importance of studying AgNP bioreactivity in the presence of lung secretions to accurately represent the likely effects of inhalation.

\section{P140 BPIFB1/LPLUNC1 IS A NOVEL MARKER FOR THE BRONCHIOLISED EPITHELIUM IN IPF}

${ }^{1} \mathrm{CD}$ Bingle, ${ }^{1} \mathrm{~B}$ Araujo, ${ }^{2}$ WA Wallace, ${ }^{1} \mathrm{HM}$ Marriott, ${ }^{2} \mathrm{~N}$ Hirani, ${ }^{1} \mathrm{~L}$ Bingle; ${ }^{1}$ University of Sheffield, Sheffield, UK; ${ }^{2}$ University of Edinburgh, Edinburgh, UK

\subsection{6/thoraxjnl-2013-204457.290}

Idiopathic pulmonary fibrosis (IPF) is an irreversible and progressive lung disease with limited life expectancy after diagnosis. Histopathological studies of IPF lungs reveal the typical "Usual Interstitial Pneumonia” (UIP) pattern, with epithelial hyperplasia, areas of scarring with fibroblast foci and characteristic morphological abnormalities, including bronchiolization of alveolar ducts and honeycomb cysts. Although it seems likely that bronchiolar abnormalities are caused by changes in epithelial cell differentiation, specific markers of this process remain elusive. By analysis of published array data sets from IPF patients, we identified BPIFB1/LPLUNC1 as a potential candidate marker for the disease. Indeed, in the largest published study the gene was the most differentially expressed transcript. This putative innate defence protein is normally expressed in the airway submucosal glands and in a population of MUC5A/C positive goblet cells in the upper airways. Analysis of lung tissue from UIP revealed strong staining of BPIFB1/LPLUNC1 within the bronchiolized epithelium lining the honeycomb cysts as well as in the mucosubstance filling these regions. MUC5B was localized to the same cells as BPIFB1/LPLUNC1 wheras the related protein, BPIFA1/SPLUNC1, was not co-expressed. This pattern of staining was not seen in other chronic lung diseases, suggesting a degree of specificity for IPF. To shed light on a temporal association of expression of these markers with fibrosis development we studied mice exposed to the pro-fibrotic agent bleomycin (Bleo). MUC5B and LPLUNC1 were co-expressed in a population of goblet cells in the airways of mice within 3-7 days of Bleo exposure, prior to the onset of fibrosis. Continued expression was seen during the development of fibrosis between 14-21 days post treatment. In contrast, in mice treated with PBS neither protein was seen (due to mouse airways being essentially free of goblet cells). Staining was absent from the fibrotic regions and the lung parenchyma, as is the case in IPF. Our data show that the ectopic expression seen in human IPF, is mirrored by that seen in the fibrotic mouse model. Furthermore it suggests that BPIFB1/LPLUNC1 may be worthy of further study as potential marker for the disease.

\section{P141 DIFFERENTIAL EXPRESSION OF CONVENTIONAL AND INHIBITORY VEGFA ISOFORMS IN NORMAL AND FIBROTIC FIBROBLASTS-A POTENTIAL ROLE IN IPF PATHOGENESIS?}

${ }^{1} \mathrm{SL}$ Barratt, ${ }^{1} \mathrm{~T}$ Blythe, ${ }^{1} \mathrm{C}$ Jarrett, ${ }^{1} \mathrm{~K}$ Ourradi, ${ }^{2} \mathrm{~T}$ Maher, ${ }^{1} \mathrm{Gl}$ Welsh, ${ }^{3} \mathrm{DO}$ Bates, ${ }^{1} \mathrm{AB}$ Millar; ${ }^{1}$ Academic Respiratory Unit, School of Clinical Sciences, Bristol University, Bristol, UK; ${ }^{2}$ Imperial College, London, UK; ${ }^{3}$ Microvascular Research Laboratories, Bristol University, Bristol, UK

\subsection{6/thoraxjnl-2013-204457.291}

Introduction Vascular endothelial growth factor (VEGFA) has been implicated in the pathogenesis of Idiopathic Pulmonary Fibrosis (IPF). Two families of endogenous isoforms exist formed by alternative splicing of mRNA transcripts: the conventional potent angiogenic and mitogenic isoforms $\left(\mathrm{VEGF}_{\mathrm{xxx}} \mathrm{a}\right.$ family) and the $\mathrm{VEGF}_{\mathrm{xxx}} \mathrm{b}$ family that is thought to have contrasting inhibitory functions.

Hypothesis We hypothesise that differential expression of $\mathrm{VEGF}_{\mathrm{xxx}} \mathrm{a}$ and $\mathrm{VEGF}_{\mathrm{xxx}} \mathrm{b}$ isoforms by fibroblasts may influence the development of IPF.

Methods Normal (NF) and fibrotic fibroblasts (FF) (from patients with proven UIP) were extracted from lung samples using the explant method. The expression of $\mathrm{VEGF}_{\mathrm{xxx}} \mathrm{a}$ and VEGF $_{x x x}$ b by NF and FF was analysed at the mRNA level by RT-PCR and quantified by qPCR. Protein expression was determined by western blotting (WB) and ELISA. We sought to establish a potential functional effect of recombinant $\mathrm{VEGF}_{165} \mathrm{a}$ and $\mathrm{VEGF}_{165} \mathrm{~b}$ proteins on fibroblasts by assessing the expression of a) the extracellular matrix (ECM) protein fibronectin and b) $\alpha$ SMA, a marker of myofibroblast differentiation.

Results Both NF and FF expressed VEGF $_{x x x} a$ and $V_{E G F} F_{x x x} b$ isoforms at the mRNA level as determined by RT-PCR with confirmation by direct sequencing. There was no statistical difference in total VEGF mRNA expression between the two cell types by qPCR ( $\mathrm{p}=0.9307, \mathrm{NF} \mathrm{n}=5, \mathrm{FF} \mathrm{n}=6$ ), but $\mathrm{FF}$ expressed significantly more $V_{E G F} 165$ mRNA than NF $(\mathrm{p}=0.05, \mathrm{NF} \mathrm{n}=5, \mathrm{FF} \mathrm{n}=6)$. Total VEGF protein expression was significantly increased in FF (mean expression 